\title{
QUEEN'S
UNIVERSITY
BELFAST
}

\section{Statin use after diagnosis is associated with an increased survival in esophageal cancer patients: a Belgian population-based study}

Lacroix, O., Couttenier, A., Vaes, E., Cardwell, C. R., De Schutter, H., \& Robert, A. (2019). Statin use after diagnosis is associated with an increased survival in esophageal cancer patients: a Belgian population-based study. Cancer Causes \& Control: an international journal of studies of cancer in human populations, 30(4), 385393. https://doi.org/10.1007/s10552-019-01149-3

Published in:

Cancer Causes \& Control: an international journal of studies of cancer in human populations

Document Version:

Peer reviewed version

Queen's University Belfast - Research Portal:

Link to publication record in Queen's University Belfast Research Portal

Publisher rights

Copyright 2019, Springer.

This work is made available online in accordance with the publisher's policies. Please refer to any applicable terms of use of the publisher.

\section{General rights}

Copyright for the publications made accessible via the Queen's University Belfast Research Portal is retained by the author(s) and / or other copyright owners and it is a condition of accessing these publications that users recognise and abide by the legal requirements associated with these rights.

Take down policy

The Research Portal is Queen's institutional repository that provides access to Queen's research output. Every effort has been made to ensure that content in the Research Portal does not infringe any person's rights, or applicable UK laws. If you discover content in the Research Portal that you believe breaches copyright or violates any law, please contact openaccess@qub.ac.uk. 
TITLE: Statin use after diagnosis is associated with an increased survival in esophageal cancer patients: A Belgian population-based study

3 Olivia Lacroix ${ }^{a}$, Alexandra Couttenier ${ }^{a}$, Evelien Vaes $^{b}$, Chris $^{\text {R. }}$ Cardwell $^{c}$, Harlinde De

$5{ }^{a}$ Université catholique de Louvain, Institut de recherche expérimentale et clinique, Pôle 6 d'épidémiologie et biostatistique, Brussels, Belgium

$7 \quad$ Research Department, Belgian Cancer Registry, Brussels, Belgium.

$8{ }^{c}$ Cancer Epidemiology and Health Services Research Group, Centre for Public Health, 9 Queen's University Belfast, Belfast, Northern Ireland, United Kingdom

\section{Corresponding author:}

12 Olivia Lacroix

13 Université catholique de Louvain (UCL), Institut de recherche expérimentale et clinique 14 (IREC), Epidémiologie et Biostatistique (EPID)

15 Clos Chapelle-aux-champs, 30 bte B1.30.13 B-1200 Bruxelles, Belgique.

16 E.mail: olivia.lacroix@uclouvain.be

17 ORCID : 0000-0003-2774-2226 
PURPOSE:

4 Preclinical studies have shown that statins reduce proliferation in esophageal cancer. Three recent observational studies have shown encouraging results but suffered from limitations. This work aimed to assess at the Belgian population level whether statin usage was associated with a decreased mortality in esophageal cancer patients.

METHODS:

9 We conducted an observational, population-based study by linking data of the Belgian Cancer Registry (BCR) with medical claims data coming from health insurance companies and mortality records collected by regional governments for patients diagnosed with esophageal cancer between 2004 and 2014. Using time-dependent Cox regression models, hazard ratios (HRs) and 95\% confidence intervals (CI) for overall and cancer-specific mortality were

14 calculated.

RESULTS:

Of 6,238 patients with stage I-III esophageal cancer, post-diagnostic use of statins was found

17 in $1,628(26 \%)$ patients. Statins use after diagnosis was associated with a reduction in overall 18 mortality (adjusted HR=0.84, 95\%CI: [0.77; 0.92]) and cancer specific mortality (adjusted $19 \mathrm{HR}=0.87,95 \% \mathrm{CI}$ : [0.78; 0.97]). Similar association were also seen for pre-diagnostic statin use 20 (overall mortality: adjusted $\mathrm{HR}=0.83$, 95\%CI: [0.76-0.91] and cancer-specific mortality: 21 adjusted $\mathrm{HR}=0.86,95 \% \mathrm{CI}:[0.77-0.96])$. CONCLUSIONS:

23 In this large cohort of Belgian patients with esophageal cancer, statins use after diagnosis was associated with a decreased mortality.

26 Keywords: Esophageal cancer; Statin; Survival; epidemiology; pharmacoepidemiology. 


\section{INTRODUCTION}

2 In 2015, esophageal carcinoma was the 11th most common cancer and the sixth most common cause of cancer death worldwide [1]. This cancer is characterized by two main histological subtypes --i.e. esophageal adenocarcinoma and esophageal squamous cell carcinoma-with different epidemiologic profiles. In Western countries, the predominant subtype is adenocarcinoma: mostly associated with reflux, obesity and male sex [2,3]. In the Asian highest risk region, called the esophageal cancer belt, the squamous cell subtype is more prevalent, which has mainly been associated with tobacco and alcohol consumption $[2,4,5]$.

Despite an improvement in the prognosis of this cancer, the 5-year relative survival remains poor (around 20\% in Belgium) [2,6]. Therefore, any new treatment modalities that would improve survival would be of significant importance.

Statins are lipid lowering agents used for primary and secondary prevention of atherosclerotic cardiovascular diseases [7]. As cardiovascular diseases remain a leading cause of morbidity and mortality, statins are amongst the most widely used medications with more than 200 million people taking these drugs [7-9].

Statins reduce low-density lipoprotein (LDL) by selectively inhibiting the 3-hydroxy-3methylglutaryl coenzyme A (HMG-CoA) reductase, the initial enzyme involved in the cholesterol biosynthesis. This inhibition also affects the production of isoprenoid intermediates, which play an important role in many physiological processes [10]. As carcinogenesis is included in those processes, it has been assumed that the pleiotropic effects of statins could be extended to the field of oncology [10].

In a more specific way, several preclinical studies have shown that statins reduce esophageal cancer cells growth and proliferation, and increased apoptosis [11-14]. More recently, three epidemiological studies have shown some interesting results. The first one demonstrated a large significant reduction in esophageal cancer-specific and overall mortality, limited to the adenocarcinoma subtype [15]. The second study found little evidence of a reduction in mortality with statins use after diagnosis and showed no differences between histologic subtypes [16]. However, important prognostic factors, such as cancer stage were not considered in these two studies. The third study showed that statin use was associated with an increase in survival but possible reverse causation was pointed by Authors [17].

31 In the present study, we aimed to investigate if statin use after diagnosis was associated with cancer-specific and overall mortality of esophageal cancer at the Belgian population level. 
1 Based on previous studies, we hypothesized that statin use after diagnosis might be associated 2 with a decreased mortality for this cancer type.

3 


\section{MATERIAL AND METHODS}

\subsection{Study design}

As described in another report, our data set resulted from the linkage of several databases [18]. First, the cancer cases were derived from the Belgian cancer registry's (BCR) database. The BCR is a population-based cancer registry covering more than $95 \%$ of the Belgian population since 2004. A complete description of the BCR role, objective and data flow is available elsewhere [19]. Vital status was provided by the crossroads bank for social security (CBSS). Diagnostic and therapeutic procedures stemmed from the Intermutualistic agency (IMA). Both vital status and therapeutic procedures were linked to the BCR database using the patient's specific national social security identification number (SSIN). Regional authorities provided causes of death data that were probabilistically coupled to the BCR database.

Patients with stage I to III esophageal adenocarcinoma or squamous cell carcinoma diagnosed between January 1, 2004 and December 31th, 2014 were identified from the BCR database. Patients with prior history of cancer (except for non-melanoma skin cancer) were excluded. Further exclusion criteria referred to individuals not residing in Belgium at the time of diagnosis, with an uncertain date of diagnosis, with no national social security identification number (SSIN), lost to follow up at the date of cancer incidence, or missing from the medical claims (IMA) database. In the main analysis patients who died in the first six months after their diagnosis were also excluded as drug use during this time is unlikely to exert an effect on cancer death.

\subsection{Exposure data}

In Belgium, five different types of statins are available for clinical use: simvastatin, fluvastatin, pravastatin, rosuvastatin and atorvastatin. Therefore, statin use was defined as a prescription of any of those subtypes. Post-diagnostic use of statins was defined as a time varying covariate in order to avoid immortal-time bias. Patients were considered non-users before their first postdiagnostic statins prescription. Then, they were considered exposed after a 6-months lag period until the end of follow-up. The 6-months lag period was used to remove prescriptions occurring prior to death as they may reflect end of life treatment, thus avoiding reverse causation bias.

In sensitivity analyses, pre-diagnostic use of statins was defined as a dispense of any of the statins mentioned above recorded from 1 month before diagnosis. 
2 Patient- and tumor-related covariates included gender, age at diagnosis, comorbidities, stage, morphology (adenocarcinoma and squamous cells carcinoma) and cancer treatments at 6 months (surgery/radiotherapy/chemotherapy).

Comorbidities in the year prior to diagnosis were derived from claims data including in-and outpatient dispensed medication, according to a previously described methodology $[18,20]$.

7 Concomitant medications were defined as the presence of at least one prescription given in the same time-period than statins for the following drugs: Beta-blocker, angiotensin conversing inhibitor (ACEi) or angiotensin receptor blocker (ARB), metformin and insulin.

\subsection{Outcome measures}

The primary outcome was overall mortality with a follow-up until July $1^{\text {st }}, 2016$. In the cancerspecific analysis, patients were followed until January $1^{\text {st }}, 2014$. Patients who died after this date were censored. Cancer specific deaths were defined as those with an underlying cause of death coded with ICD-10 C15.0-C16.9 for esophageal and gastric cancer or C26.9-C26.8 for malignant neoplasm of overlapping lesion of the digestive system.

\subsection{Statistical analysis}

The baseline characteristics of statins users and non-users were compared using Chi-square test. In post-diagnosis analyses, we investigated overall and cancer-specific mortality using Cox proportional hazard regression with time dependent exposure. Patients were followed from 6 months after cancer diagnosis until death, end of follow-up or lost to follow up.

Dose response analyses were carried out using cumulative post-diagnosis number of prescriptions or defined daily doses (DDDs) using time varying co-variables. In these analyses, statin users were first classified as non-users before the first post-diagnosis prescription, they became light users after their first prescription and heavy users at the date when they tread over the $12^{\text {th }}$ prescription or the $365^{\text {th }}$ DDD.

The main analysis was also performed to compare each type of statins separately to statins nonusers.

Subgroup analyses were conducted by histological subtype, sex, and cancer treatment within 6 months after diagnosis. In secondary analyses, we investigated the association between prediagnostic statin use in the year prior to diagnosis without excluding those patients with less 
1 than 6 months of follow-up after diagnosis. A simplified analysis using statins prescriptions in

2 the first 6 months after esophageal cancer diagnosis in patients with more than 6 months of

3 follow-up was also conducted. This kind of analysis allows controlling for immortal time bias

4 without using time-varying covariates [21]. Indeed, statin use is only assessed within the 6

5 months after diagnosis in 6 months' survivors, therefore there is no survival bias.

6 All analyses were adjusted for sex, age, year of diagnosis, comorbidities, cancer treatment

7 within 6 months after diagnosis, and cancer histology. An adjusted analysis was also conducted

8 with concomitant medication used (angiotensin-converting enzyme inhibitors, angiotensin 2-

9 receptor blockers, beta-blockers, insulin and metformin) as time-varying covariate.

10 Sensitivity analyses regarding the length of the lag were also conducted. First, the main analysis

11 was reproduced without lag (and without excluding deaths after cancer diagnosis) then, with a

12 3- months lag (excluding deaths in the three months after diagnosis) and finally with a 12-

13 months lag (excluding deaths in the first year after diagnosis).

14 In all analyses, censoring was conducted at 5 years after diagnosis. All analyses were carried 15 out with SAS Enterprise Guide statistical release 9.3 software. 


\subsection{Patient cohort}

3 A total of 6,238 patients who met the inclusion criteria were identified from the BCR database

4 with a diagnosis of esophageal cancer between 2004 and 2014. In cancer-specific analyses 71

5 patients were excluded because they had no cause of deaths.

6 A flowchart showing the number of patients included in each analysis is provided in Figure 1.

7

7,573 were excluded for at least one reason:

- Stage IV or missing: $\mathrm{n}=6,422$

- Multiple tumor: $\mathrm{n}=2,054$

- Missing from hospital database: $\mathrm{n}=218$

- Incidence date $=$ date of death: $\mathrm{n}=22$

- Incidence date $=$ date of lost to follow up: $n=13$

\section{Excluded because $<6$ months of follow up \\ Overall analyses $\mathrm{n}=1,004$ \\ Cancer specific analyses $\mathrm{n}=1,092$}

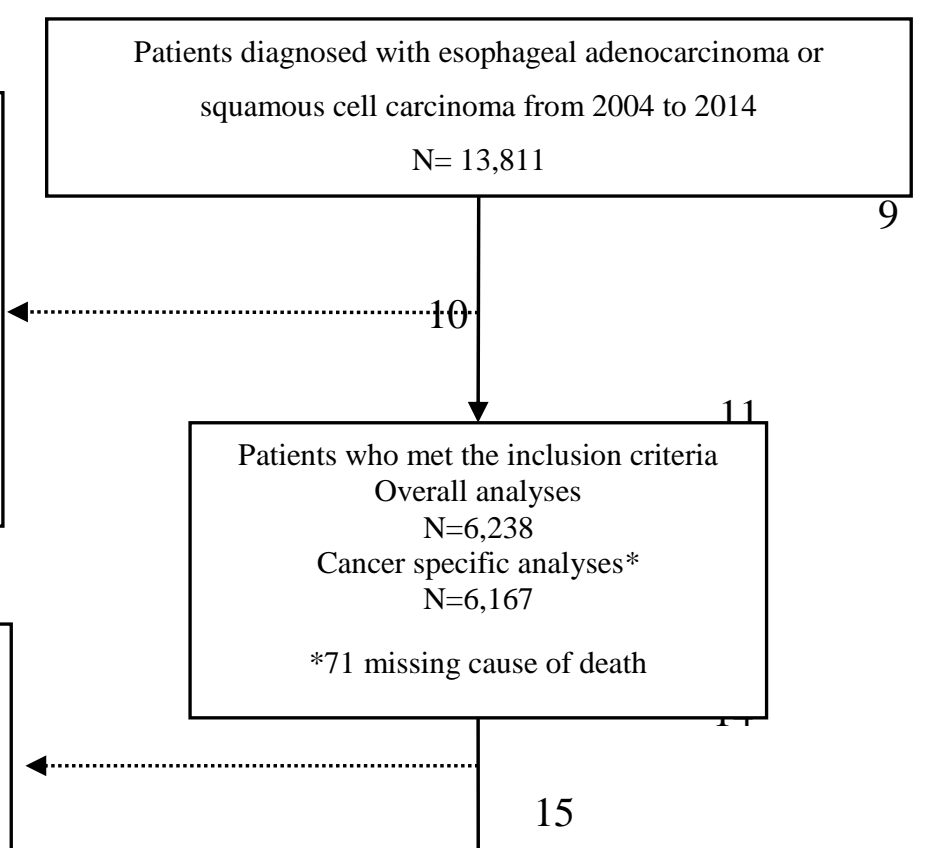

17

18

19

20

21

22 Overall analyses

$\mathrm{N}=1,628$

Cancer-specific analyses $\mathrm{N}=1,572$
Post-diagnosis statins users
Post-diagnosis statins non-users Overall analyses $\mathrm{N}=3,606$

Cancer-specific analyses $\mathrm{N}=3,503$

Figure 1. Flow of study participants 
1 From the 5,234 patients included in the overall analysis, 3,015 (57.6\%) died from any cause 2 within 5 years after diagnosis. In cancer-specific analysis 2,003 (78.2\%) deaths were considered 3 due to esophageal cancer. The median follow up time was 2.39 years (IQR: 1.22-4.85). The 4 median time to the first post diagnosis prescription was 43 days (Interquartile range (IQR): 175 121.5).

6 From the 6,238 patients who met the inclusion criteria, 1,939 (31.1\%) were statins users in the 7 year prior to their diagnosis. After diagnosis, 391 (20.1\%) patients who were previous users 8 stopped using statins.

9 Patients' characteristics by post diagnostic statin use are shown in Table I. Post diagnosis statin 10 users were older, diagnosed more recently, with mainly adenocarcinoma subtype and had more 11 associated comorbidities. They also tended to use more concomitant medications and 12 underwent less surgery/chemotherapy/RT treatments for their cancer. Similar observations 13 were made when comparing pre-diagnosis statin users to non-users (not shown). 
Table 1: Statin use before and after esophageal cancer diagnosis, Belgium, 2004-2014

\begin{tabular}{|c|c|c|c|}
\hline \multirow[b]{2}{*}{ Characteristics } & \multicolumn{3}{|c|}{ Statin after diagnosis $(\mathrm{N}=5,234) \ddagger$} \\
\hline & $\begin{array}{c}\text { Users } \\
(\mathrm{n}=1,628)\end{array}$ & $\begin{array}{c}\text { Non-users } \\
(\mathrm{n}=3,606)\end{array}$ & $p$ value \\
\hline Age - (yrs.) & & & $<0.001$ \\
\hline Mean \pm SD & $68 \pm 9$ & $64 \pm 12$ & \\
\hline Age Category --n (\%) & & & $<0.001$ \\
\hline$<60$ & $327(20)$ & $1,362(38)$ & \\
\hline $60-69$ & $601(37)$ & $1,083(30)$ & \\
\hline $70-79$ & $521(32)$ & $782(22)$ & \\
\hline $80-89$ & $174(11)$ & $362(10)$ & \\
\hline$\geq 90$ & $5(0)$ & $17(0)$ & \\
\hline $\operatorname{Sex}-\mathbf{n}(\%)$ & & & 0.19 \\
\hline Men & $1,262(78)$ & $2.735(76)$ & \\
\hline Year of diagnosis-n (\%) & & & $<0.001$ \\
\hline$<2009$ & $522(32)$ & $1,540(43)$ & \\
\hline$\geq 2009$ & $1,106(68)$ & $2,066(57)$ & \\
\hline Grade of differentiation-n $(\%)$ & & & 0.10 \\
\hline Poorly & $528(32)$ & $1,200(33)$ & \\
\hline Moderately & $581(36)$ & $1,352(38)$ & \\
\hline Well & $251(15)$ & $467(13)$ & \\
\hline Unknown/missing & $268(17)$ & $587(16)$ & \\
\hline Morphology---n (\%) & & & $<0.001$ \\
\hline Squamous cell & $513(32)$ & $1,385(38)$ & \\
\hline Adenocarcinoma & $1,115(68)$ & $2,221(62)$ & \\
\hline Combined stage $-\mathbf{n}(\%)$ & & & $<0.001$ \\
\hline I & $565(35)$ & $987(27)$ & \\
\hline II & $476(29)$ & $1,061(29)$ & \\
\hline III & $587(36)$ & $1.558(43)$ & \\
\hline Cardiovascular disease $-\mathrm{n}(\%)$ & & & $<0.001$ \\
\hline Yes & $1,158(71)$ & $1,429(40)$ & \\
\hline Respiratory diseases-n (\%) & & & 0.002 \\
\hline Yes & $134(8)$ & $215(6)$ & \\
\hline Diabetes-n $(\%)$ & & & $<0.001$ \\
\hline Yes & $372(23)$ & $346(10)$ & \\
\hline \multicolumn{4}{|l|}{ Concomitant medication use $-\mathrm{n}(\%)$} \\
\hline ACEi or ARB & $863(53)$ & $884(25)$ & $<0.001$ \\
\hline Beta-blocker & $1,165(72)$ & $1,720(48)$ & $<0.001$ \\
\hline Metformin & 303 (19) & $270(7)$ & $<0.001$ \\
\hline Insulin & $669(41)$ & $1,100(30)$ & $<0.001$ \\
\hline Cancer therapy at 6 months $-n(\%)$ & & & $<0.001$ \\
\hline Surgery with CT or RT & $467(29)$ & $1,161(32)$ & \\
\hline Surgery alone & $463(28)$ & $898(25)$ & \\
\hline CT or RT without surgery & $477(29)$ & $1,182(33)$ & \\
\hline No treatment & $221(14)$ & $365(10)$ & \\
\hline
\end{tabular}




\subsection{Post-diagnosis statin use}

In post-diagnosis analyses, statin use was independently associated with a significant $16 \%$ decrease in overall mortality (adjusted $\mathrm{HR}=0.84$; 95\% CI: $0.77-0.92$ ) and a $13 \%$ decrease in cancer-specific mortality (adjusted HR=0.87; 95\% CI: 0.78-0.97) (Table II).

No significant dose-response associations were observed with either the number of prescriptions or the number of DDDs for overall mortality. In cancer-specific analysis, despite the apparent decrease in mortality from light to heavy users when considering DDDs, the mortality difference between those two groups did not reach a significant level (>365 versus $\leq 365$ DDDs, HR=0.93; 95\% CI: 0.76-1.15).

Post-diagnosis use of each type of statin except rosuvastatin and fluvastatin was associated with a decreased overall and cancer-specific mortality compared to statin non-users (Supplementary table I). 
Table 2. Post diagnosis statin use and overall or cancer-specific mortality in patients with esophageal cancer between 2004-2014 in Belgium

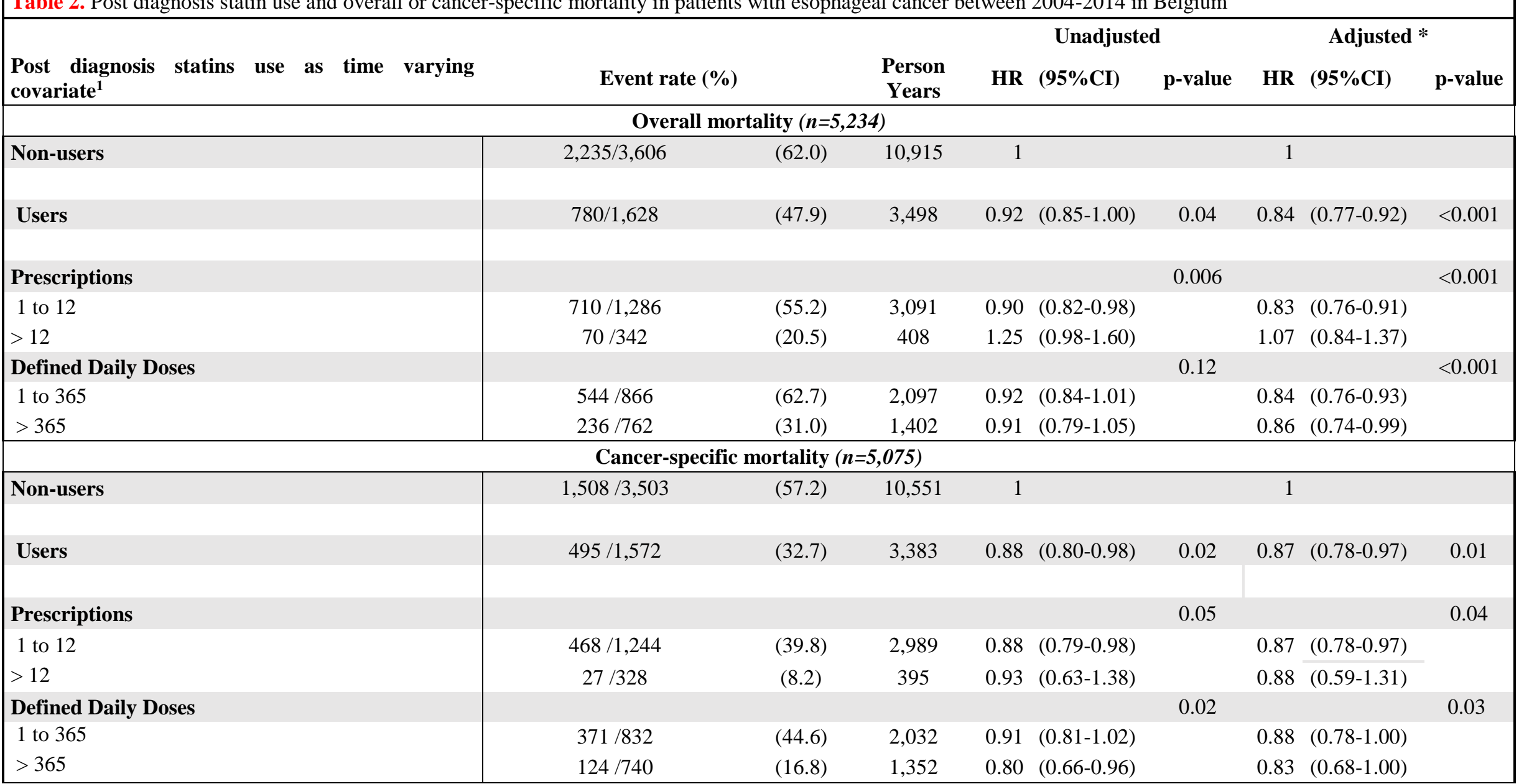

*Adjusted for age (continuous), sex, year of diagnosis (<2009 and $\geq 2009)$, stage, cancer treatment, morphology (adenocarcinoma or squamous cell carcinoma) and comorbidities (cardiovascular, diabetes and respiratory)

${ }^{1}$ Analyses included a lag of 6 months in individuals living more than 6 months

p-value are for likelihood ratio test comparing model with and without the specific variable 


\subsection{Sensitivity and subgroup analysis}

Analysis of post-diagnosis statin use gave similar results after additional adjustment for concomitant medications in overall mortality (adjusted $\mathrm{HR}=0.83$; 95\% CI: 0.76-0.91) and cancer-specific mortality (adjusted HR=0.86; 95\%CI: 0.77-0.96).

Also, reassuringly, the simplified analysis of statin use in the 6 months following diagnosis gave similar results as the time varying analysis for both overall (adjusted HR=0.86; 95\%CI: 0.79-0.93) and cancer-specific mortality (adjusted $\mathrm{HR}=0.86$; 95\%CI: 0.78-0.96).

Statins use before diagnosis was associated with a $16 \%$ decrease in overall mortality (adjusted $\mathrm{HR}=0.84$; 95\% CI: $0.78-0.90)$ and $18 \%$ decrease in cancer-specific mortality (adjusted $\mathrm{HR}=0.82$; 95\%CI: 0.75-0.90).

Sensitivity analyses regarding the length of the lag period are shown in table III. The association between post-diagnosis statin use and mortality, was still observed when no lag was used (overall mortality: adjusted $\mathrm{HR}=0.81 ; 95 \% \mathrm{CI}, 0.75-0.87$ and cancer-specific mortality: adjusted $\mathrm{HR}=0.81$; 95\% CI: 0.74-0.88) and with a 3 months' lag in individuals living more than 3 months (overall mortality: adjusted $\mathrm{HR}=0.83$; 95\%CI: 0.76-0.90 and cancer-specific mortality: adjusted $\mathrm{HR}=0.83$; 95\% CI: 0.75-0.91). When a 12 months' lag in individuals living more than 1 year was applied, the association remained similar for overall mortality (adjusted HR=0.87; 95\%CI: 0.79-0.97) but did not reach significance in cancer-specific analysis (adjusted $\mathrm{HR}=0.94$; 95\%CI: 0.83-1.08).

In subgroup analysis, interaction tests did not show a difference between statin use and mortality by histologic subtypes (overall mortality: $\mathrm{p}$ for interaction $=0.60$ and cancer-specific mortality: $\mathrm{p}$ for interaction $=0.89$ ), sex (overall mortality: $\mathrm{p}$ for interaction $=0.54$ and cancer-specific mortality: $\mathrm{p}$ for interaction $=0.60$ ) or cancer treatment in the six months after diagnosis (Supplementary figure I). 


\begin{tabular}{|c|c|c|c|c|c|c|c|c|c|c|}
\hline \multirow[b]{2}{*}{ Medication usage } & & \multirow{2}{*}{\multicolumn{2}{|c|}{ Event rate (\%) }} & \multirow[b]{2}{*}{ Person Years } & \multicolumn{3}{|c|}{ Unadjusted } & \multicolumn{3}{|c|}{ Adjusted * } \\
\hline & & & & & HR & $(95 \% \mathrm{CI})$ & $\mathbf{p}$ & HR & $(95 \% \mathrm{CI})$ & $\mathbf{p}$ \\
\hline \multicolumn{11}{|c|}{ Post diagnosis statins use as time varying covariate } \\
\hline \multicolumn{11}{|c|}{ Overall mortality } \\
\hline \multirow[t]{2}{*}{ without $\operatorname{lag}^{1}(n=6,238)$} & Non-users & $2,951 / 4,303$ & (68.6) & 10,298 & 1 & & & 1 & & \\
\hline & Users & $1,066 / 1,935$ & (55.1) & 4,389 & 0.89 & $(0.83-0.95)$ & $<0.001$ & 0.81 & $(0.75-0.87)$ & $<0.001$ \\
\hline \multirow[t]{2}{*}{ 3months $\operatorname{lag}^{2}(n=5,794)$} & Non-users & $2,644 / 4,006$ & $(66.0)$ & 10,699 & 1 & & & 1 & & \\
\hline & Users & $930 / 1,788$ & $(52.0)$ & 3,923 & 0.91 & $(0.84-0.98)$ & $<0.01$ & 0.83 & $(0.76-0.90)$ & $<0.001$ \\
\hline \multirow[t]{2}{*}{1 year $\operatorname{lag}^{3}(n=4,242)$} & Non-users & $1,489 / 2,881$ & (51.7) & 10,933 & 1 & & & & & \\
\hline & Users & $535 / 1,361$ & (39.3) & 2,756 & 0.95 & $(0.86-1.05)$ & 0.30 & 0.87 & $(0.79-0.97)$ & 0.01 \\
\hline \multicolumn{11}{|c|}{ Cancer-specific mortality } \\
\hline \multirow[t]{2}{*}{ without $\operatorname{lag}^{1}(n=6,167)$} & Non-users & $2,033 / 4,266$ & $(47.7)$ & 9,979 & 1 & & & 1 & & \\
\hline & Users & $682 / 1,901$ & $(35.8)$ & 4,249 & 0.83 & $(0.77-0.91)$ & $<0.001$ & 0.81 & $(0.74-0.88)$ & $<0.001$ \\
\hline \multirow[t]{2}{*}{ 3months $\operatorname{lag}^{2}(n=5,669)$} & Non-users & $1,814 / 3,935$ & (46.1) & 10,361 & 1 & & & 1 & & \\
\hline & Users & $590 / 1,734$ & $(34.0)$ & 3,795 & 0.85 & $(0.78-0.94)$ & $<0.001$ & 0.83 & $(0.75-0.91)$ & $<0.001$ \\
\hline \multirow[t]{2}{*}{1 year $\operatorname{lag}^{3}(n=4,081)$} & Non-users & $962 / 2,763$ & (34.8) & 10,544 & 1 & & & 1 & & \\
\hline & Users & $328 / 1,318$ & (24.9) & 2,668 & 0.92 & $(0.81-1.05)$ & 0.21 & 0.94 & $(0.83-1.08)$ & 0.39 \\
\hline \multirow{2}{*}{\multicolumn{11}{|c|}{$\begin{array}{l}\text { *Adjusted for age (continuous), sex, year of diagnosis ( }<2009 \text { and } \geq 2009 \text { ), stage, cancer treatment, morphology (adenocarcinoma or squamous cell carcinoma) and } \\
\text { comorbidities (cardiovascular, diabetes and respiratory) }\end{array}$}} \\
\hline & & & & & & & & & & \\
\hline \multicolumn{11}{|c|}{${ }^{2}$ Analyses include all individuals with more than 3 months of follow-up } \\
\hline \multicolumn{11}{|c|}{${ }^{3}$ Analyses include all individuals with more than 1 year of follow-up } \\
\hline
\end{tabular}




\section{DISCUSSION}

2 In this large, population based cohort of 5,234 patients with incident esophageal cancer, post3 diagnosis statins use was associated with a $16 \%$ decrease in overall mortality and a $13 \%$ decrease in cancer-specific mortality. Similar associations were seen when investigating pre-diagnosis statin use. However, there were no dose-response relationships when considering the cumulative number of prescriptions or the cumulative number of DDDs and there was no apparent difference between the two main histological subtypes.

8 Our findings match with previous results regarding statins use and cancer outcome. Indeed, statins 9 have been consistently associated with a decreasing mortality in cancer [22,23]. In gastrointestinal 10 cancer particularly, meta-analyses of observational studies have associated statins use with a 11 decreased mortality in colorectal cancer [22,24]. Statins have also been tested in phase II and III 12 randomized trials for advanced gastric cancer with no improvement seen in survival outcome [25,26]. 13 Those studies, however, focused on metastatic patients not amenable for curative resection.

14 The anti-tumor effect of statins is thought to occur through the inhibition of the mevalonate pathway 15 as cancer cells are known to be highly dependent on the mevalonate pathway metabolites. More 16 specifically cholesterol, an important component of cell membranes, is mainly obtained by cancer 17 cells through this pathway [27]. Also, small guanosines triphosphatases (GTPases) involved in 18 carcinogenesis such as Ras, Rho, Rab, Arf and Ran are dependent on prenylation, one of the steps in 19 the mevalonate pathway [27].

20 In esophageal adenocarcinoma cells specifically, simvastatin, lovastatin, atorvastatin and pravastatin, 21 which are subtypes of the statin family, have been associated with inhibition of cancer cell 22 proliferation [11,12]. Simvastatin and pravastatin have also been associated with an increased 23 apoptosis through the inhibition of prenylation step [11]. In esophageal adenocarcinoma a decrease 24 in the oncoprotein Ras activity was observed after treatment with simvastatin [11]. This oncoprotein 25 is known to be highly correlated to the mevalonate pathway [27]. Simvastatin and Atorvastatin were 26 also involved in the inhibition of metastasis [12].

27 In esophageal squamous cell carcinoma, only lovastatin was studied. In those cancerous cells, 28 lovastatin decreased cancer cells growth by inhibiting the upregulated mevalonate pathway, thus 29 leading to a decreasing activity of the oncoprotein Ras [13].

30 Consistently, simvastatin, pravastatin and atorvastatin were the three statins subtypes available in 31 Belgium that were associated with lower overall and esophageal cancer specific mortality in the 32 present study, thereby aligning with the pre-clinical studies. 
1 At the population level, three studies have previously investigated statin use after esophageal cancer 2 diagnosis.

3 The first used the United Kingdom General Practice Research Database (GPRD), the world's largest 4 electronic database of prospective demographic, lifestyle and medical data in primary care [15]. This 5 study found a $38 \%$ decreased risk of esophageal cancer-specific mortality and a $37 \%$ decrease risk of 6 overall mortality in post-diagnosis statin users in a population of 4,445 patients [15]. However, their 7 conclusion relied on unlagged analysis, without adjustment on cancer stage, and on a substantial 8 amount of missing data for treatment. In comparison, and even with unlagged prescription, the 9 magnitude of the effect was lower in our analysis when considering esophageal cancer-specific mortality. In overall mortality however, our findings were similar: they showed a $15 \%$ reduction of 11 mortality, and our results suggest a $16 \%$ reduction.

12 The differences in cancer specific analysis could be explained by differences in cancer-specific deaths 13 definition. They considered as cancer specific deaths, those in which esophageal cancer was listed in 14 part one of death certificate while we also include gastric cancer deaths (C15) and malignant 15 neoplasm of other and ill-defined digestive organs (C26).

16 Pre-diagnosis statin use was also investigated as a prescription for a minimum of two months between 176 and 18 months. The authors show a reduction of $14 \%$ in overall mortality and a non-significant $9 \%$ 18 reduction for cancer specific mortality. In comparison, we found a $16 \%$ reduction of overall mortality 19 and an $18 \%$ reduction for cancer-specific mortality. Again, our definition of cancer-specific deaths 20 allowed us to have more events. In addition, in our study pre-diagnosis users were defined as those 21 with a prescription of any kind of statin between 1 and 12 months before the diagnosis.

22 The second study used the Scottish Cancer Registry database and was based upon 1,921 newly 23 diagnosed esophageal cancer patients [16]. The authors concluded on little evidence of a reduction 24 in esophageal cancer-specific mortality while their main analyses showed a non-significant $7 \%$ reduction of mortality. Statins use before diagnosis was, however associated with a $12 \%$ reduction in overall mortality and an $11 \%$ cancer specific mortality. In comparison, our effect size was bigger but we also have included more patients. Moreover, the authors were able to adjust their analyses for deprivation, but were unable to adjust for cancer stage.

29 In a large cohort study including 11,750 patients diagnosed with esophageal cancer during 15 30 consecutive years in the United States, statin use after esophageal cancer diagnosis was associated 31 with a $10 \%$ decrease in mortality [17]. As the two-previous study, they included all stage patients, and so metastatic patients with a poor survival were also included. That can explain the smaller effect 
1 size and differences in analysis with different lag. Indeed, for each lagged analysis they suppressed 2 dead patients according to the lag duration. For example, in the 6-months lag analysis, suppressed 3 patients with less than 6 months of follow-up, should be mainly stage IV patients.

4 It is interesting to note that despite little methodological differences with the three previous studies, 5 our results are headed in the same direction.

6 Our result may have some limitations. First, some potentially important confounders like e.g. body 7 mass index (BMI), smoking, alcohol and socioeconomic status were missing from our database.

$8 \mathrm{BMI}$ is a recognized risk factor for esophageal adenocarcinoma. Moreover, in some studies, BMI was 9 also associated with survival outcome in esophageal cancers patients and this association might be 10 modified by smoking status [28]. However, distinction should be made considering the time of BMI 11 assessment. If a high BMI is considered as a risk factor for adenocarcinoma, weight loss before 12 surgery in esophageal cancers and low BMI in the first 6 months after surgery in adenocarcinoma 13 were associated with poor prognosis [29,30]. This phenomenon, well known in cardiovascular 14 literature, is called the obesity paradox [31]. Therefore, healthier patients (that is patients with a 15 higher BMI in the present context) may have been prescribed selectively statin, leading to a better 16 survival: this type of bias is a selection bias also called confounding by indication. This could also be 17 one of the reasons why no dose-response was seen.

18 Socioeconomic status is a consistent risk factor for esophageal squamous cell [5]. Patients with low 19 income or lower educational level might be less health-conscious and more likely to adopt bad 20 lifestyle habits, being therefore more at risk for squamous cell carcinoma, a tumor highly associated 21 with tobacco and alcohol consumption [32]. Moreover, patients with lower socioeconomic status 22 might present with advanced stage of cancer and therefore suffer from a worse prognosis. Thus, and 23 as in the current study statin users were more likely to be diagnosed with adenocarcinoma than 24 squamous cell carcinoma, they might represent a healthier population with higher socioeconomic status. This could contribute to spurious association between statin use and an improved survival.

26 Prevalent user bias is frequently cited in pharmacoepidemiological studies and restricting analysis to 27 the subset of new users is a way to avoid it [33]. In our study, only $23 \%$ of post-diagnosis users were 28 new users. We didn't analyze this subgroup of patients to avoid healthy users' bias as they are healthy 29 enough to initiate a preventive cardiovascular treatment after their cancer diagnosis and therefore are 30 not representative of the general population of esophageal cancer patients. 
1 One of the main strength of this study relies on the fact that we used the Belgian national cancer registry containing all cases of cancer diagnosed by hospital or laboratories. Patients were selected over 11 consecutive incidence years resulting in a large population-based set of more than 6,000 esophageal cancer cases.

5 In order to avoid immortal time bias, statin use after diagnosis was used as a time-varying variable, 6 allowing participants to be considered as non-users until they receive their first post-diagnostic statins 7 prescription. Immortal time bias is a frequent issue in observational study leading to an overestimation 8 of the studied effect [34].

9 As concomitant medications could possibly confound association with survival, we also conducted 10 an additional analysis taking into account the post-diagnosis use of metformin, insulin, beta-blockers, 11 angiotensin conversing inhibitor and angiotensin receptor blocker as time varying covariates. In a 12 reassuring way, this analysis showed results similar to the main one.

13 We also adjusted for cancer stage that is an important prognostic factor in esophageal cancer but it 14 was missing in two of the three previous studies $[15,16]$. We choose to exclude patients with stage 15 IV cancer because they have a poor 5-years relative survival $(<10 \%)$ and they are less likely to use 16 or continue statins after the cancer diagnosis.

17 Health insurance data allowed to avoid recall bias and provided precise information regarding statin 18 exposure. As in Belgium, health insurance is compulsory and covers all reimbursed medication, it 19 seems unlikely to miss statin prescriptions as they are reimbursed medication only available with 20 medical prescription. We also benefit from excellent data related to cancer incidence and 21 characteristics thanks to the Belgian population based cancer registry.

22 In summary, we used specific methods in this study to control for frequent important biases in 23 observational studies. Robust verification of cancer case and deaths was allowed by the use of a 24 nationwide cancer registry database. Moreover, it is also the first study conducted in Belgium 25 investigating the association between statin use and esophageal cancer mortality.

26 According to our results, statin use in esophageal cancer patients with good prognosis could be 27 maintained as it was not associated with poorer prognosis. However, further studies are needed to 28 explore a potential antineoplastic effect of statins before initiating such medication. We believe this 29 study could add information to the general topic of statin use and esophageal cancer progression. 


\section{5. CONCLUSION}

2 In this large, population based esophageal cancer cohort, post diagnosis statin use was associated with 3 significant decrease in overall and cancer specific mortality. Other large observational studies are 4 however needed in order to confirm these findings before conducting randomized controlled trials 5 


\section{SUPLEMENTARY MATERIAL}

\begin{tabular}{|c|c|c|c|c|c|c|c|c|}
\hline & & & & & & & & \\
\hline Medication usage & Even & $(\%)$ & Person Years & HR $(95 \% \mathrm{CI})$ & $\mathbf{p}$ & HR & $(95 \% \mathrm{CI})$ & $\mathbf{p}$ \\
\hline & & & & erall mortality & & & & \\
\hline Post diagnosis stat & use as time va & covaria & & & & & & \\
\hline statin non-users & $2,235 / 3,606$ & $(62.0)$ & 10,318 & 1 & & 1 & & \\
\hline Simvastatin & 418 / 860 & $(48.6)$ & 1,812 & $0.94(0.85-1.04)$ & 0.24 & 0.86 & $(0.77-0.96)$ & 0.007 \\
\hline statin non-users & $2,235 / 3,606$ & $(62.0)$ & 9,623 & 1 & & 1 & & \\
\hline Pravastatin & $80 / 176$ & $(45.5)$ & 401 & $0.78 \quad(0.63-0.98)$ & 0.03 & 0.71 & $(0.56-0.89)$ & 0.003 \\
\hline statin non-users & $2,235 / 3,606$ & $(62.0)$ & 9,914 & 1 & & 1 & & \\
\hline Atorvastatin & $182 / 451$ & $(40.4)$ & 987 & $0.74 \quad(0.63-0.86)$ & $<0.001$ & 0.66 & $(0.57-0.77)$ & $<0.001$ \\
\hline statin non-users & $2,235 / 3,606$ & $(62.0)$ & 9,756 & 1 & & 1 & & \\
\hline Rosuvastatin & $141 / 297$ & $(47.5)$ & 590 & $0.92 \quad(0.78-1.09)$ & 0.33 & 0.87 & $(0.73-1.04)$ & 0.13 \\
\hline statin non-users & $2,235 / 3,606$ & $(62.0)$ & 9,473 & 1 & & 1 & & \\
\hline Fluvastatin & $11 / 16$ & $(68.8)$ & 29 & $1.37 \quad(0.76-2.47)$ & 0.30 & 1.20 & $(0.66-2.17)$ & 0.55 \\
\hline & & & Canc & r-specific mortalit & & & & \\
\hline Post diagnosis stat & use as time va & covaria & & & & & & \\
\hline statin non-users & $1,508 / 3,503$ & $(43.0)$ & 9,975 & 1 & & 1 & & \\
\hline Simvastatin & 266 / 828 & $(32.1)$ & 1,761 & $0.90 \quad(0.79-1.03)$ & 0.13 & 0.88 & $(0.77-1.01)$ & 0.06 \\
\hline statin non-users & $1,508 / 3,503$ & $(43.0)$ & 9,309 & 1 & & 1 & & \\
\hline Pravastatin & $49 / 170$ & $(28.8)$ & 390 & $0.72 \quad(0.54-0.96)$ & 0.02 & 0.69 & $(0.52-0.92)$ & 0.01 \\
\hline statin non-users & $1,508 / 3,503$ & $(43.0)$ & 9,592 & 1 & & 1 & & \\
\hline Atorvastatin & 112 / 438 & $(25.6)$ & 950 & $0.69 \quad(0.57-0.84)$ & $<0.001$ & 0.67 & $(0.55-0.81)$ & $<0.001$ \\
\hline statin non-users & $1,508 / 3,503$ & $(43.0)$ & 9,439 & 1 & & 1 & & \\
\hline Rosuvastatin & $91 / 286$ & $(31.8)$ & 564 & $0.86(0.69-1.06)$ & 0.16 & 0.88 & $(0.70-1.09)$ & 0.24 \\
\hline statin non-users & $1,508 / 3,503$ & $(43.0)$ & 9,167 & 1 & & 1 & & \\
\hline Fluvastatin & $8 / 15$ & $(53.3)$ & 28 & $1.50 \quad(0.75-3.01)$ & 0.25 & 1.58 & $(0.79-3.17)$ & 0.20 \\
\hline
\end{tabular}




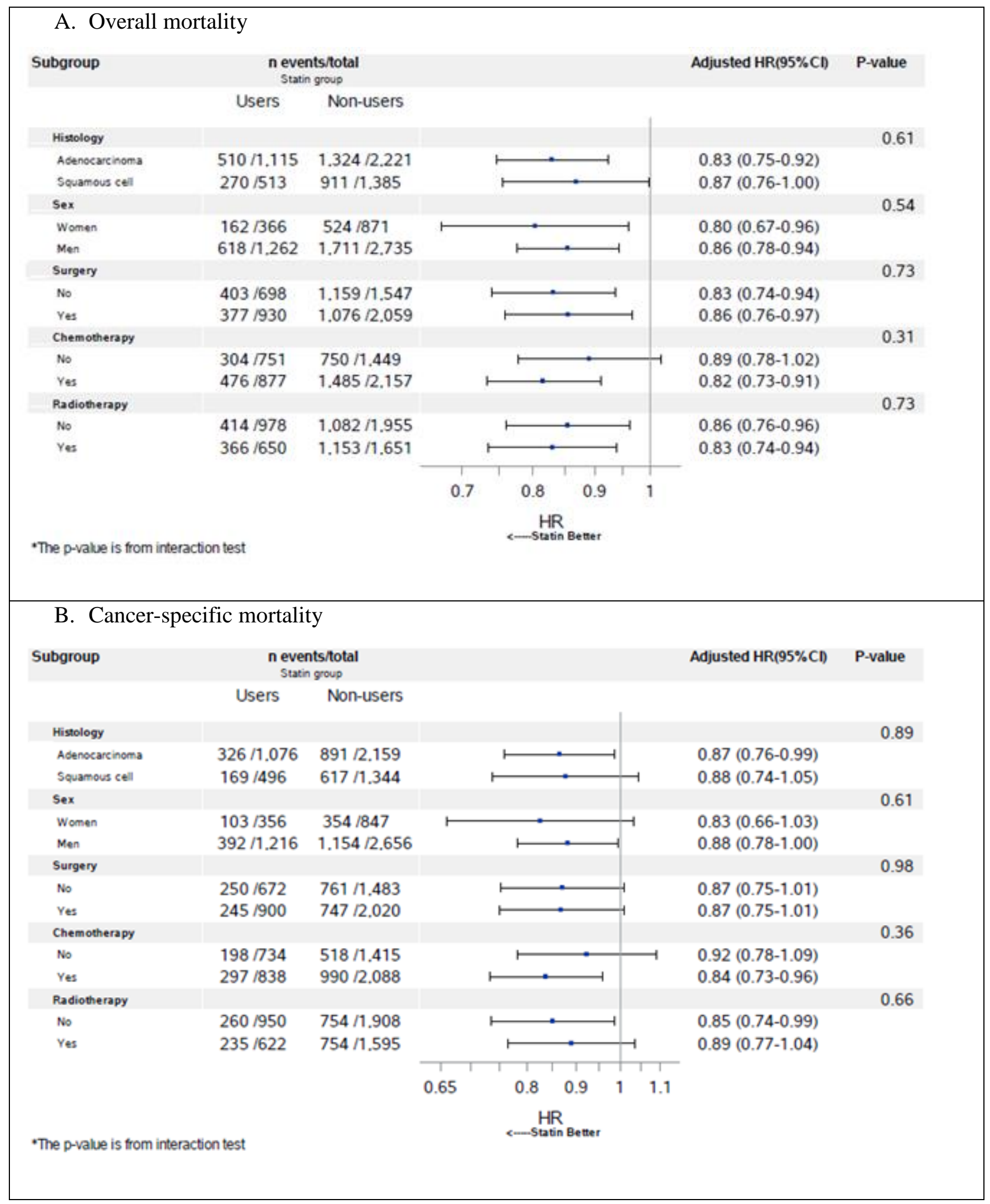

Supplementary figure 1. Subgroup analysis 


\section{ACKNOWLEDGEMENTS}

We thank for this publication the Belgian Cancer Registry collaborators who were involved in the data collection and the research team for their expertise. We also sincerely thank the Intermutualistic Agency IMA-AIM, the Belgian Crossroads bank for Social Security (BCSS) and the Belgian regions for providing the data that made this research possible. 


\section{BIBLIOGRAPHY}

1 Fitzmaurice C, Allen C, Barber RM, et al. Global, regional, and national cancer incidence, mortality, years of life lost, years lived with disability, and disability-adjusted life-years for 32 cancer groups, 1990 to 2015: A Systematic Analysis for the Global Burden of Disease Study Global Burden . JAMA Oncol 2017;3:524-48. doi:10.1001/jamaoncol.2016.5688

2 Lagergren J, Smyth E, Cunningham D, et al. Oesophageal cancer. Lancet 2017;390:2383-96. doi:10.1016/S0140-6736(17)31462-9

3 Coleman HG, Xie SH, Lagergren J. The Epidemiology of Esophageal Adenocarcinoma. Gastroenterology 2018;154:390-405. doi:10.1053/j.gastro.2017.07.046

4 Rustgi AK, El-Serag HB. Esophageal Carcinoma. N Engl J Med 2014;371:2499-509. doi:10.1056/NEJMra1314530

5 Abnet CC, Arnold M, Wei WQ. Epidemiology of Esophageal Squamous Cell Carcinoma. Gastroenterology 2018;154:360-73. doi:10.1053/j.gastro.2017.08.023

6 Anderson LA, Tavilla A, Brenner H, et al. Survival for oesophageal, stomach and small intestine cancers in Europe 1999-2007: Results from EUROCARE-5. Eur J Cancer 2015;51:2144-57. doi:10.1016/j.ejca.2015.07.026

7 Desai CS, Martin SS, Blumenthal RS. Non-cardiovascular effects associated with statins. BMJ 2014;349:1-10. doi:10.1136/bmj.g3743

8 Sirtori CR. The pharmacology of statins. Pharmacol Res 2014;88:3-11. doi:10.1016/j.phrs.2014.03.002

9 Catapano AL, Graham I, De Backer G, et al. 2016 ESC/EAS Guidelines for the Management of Dyslipidaemias. Eur Heart J 2016;37:2999-3058. doi:10.1093/eurheartj/ehw272

10 Sopková J, Vidomanová E, Strnádel J, et al. The role of statins as therapeutic agents in cancer. Gen Physiol Biophys 2017;36:501-11. doi:10.4149/gpb_2017045

11 Ogunwobi OO, Beales ILP. Statins inhibit proliferation and induce apoptosis in Barrett's esophageal adenocarcinoma cells. Am J Gastroenterol 2008;103:825-37. doi:10.1111/j.15720241.2007.01773.x

12 Sadaria MR, Reppert AE, Yu JA, et al. Statin therapy attenuates growth and malignant 
potential of human esophageal adenocarcinoma cells. $J$ Thorac Cardiovasc Surg 2011;142:1152-60. doi:10.1016/j.jtcvs.2011.08.004

13 Shi J, Zhu J, Zhao H, et al. Mevalonate pathway is a therapeutic target in esophageal squamous cell carcinoma. Tumor Biol 2013;34:429-35. doi:10.1007/s13277-012-0567-0

14 Konturek PC, Burnat G, Hahn EG. Inhibition of Barrett's adenocarcinoma cell growth by simvastatin: involvement of cox-2 and apoptosis-related proteins. J Physiol Pharmacol 2007;58:141-8.www.jpp.krakow.pl

15 Alexandre L, Clark AB, Bhutta HY, et al. Association between Statin Use after Diagnosis of Esophageal Cancer and Survival: A Population-Based Cohort Study. Gastroenterology 2016;150:854-65.e1. doi:10.1053/j.gastro.2015.12.039

16 Cardwell CR, Spence AD, Hughes CM, et al. Statin use after esophageal cancer diagnosis and survival: A population based cohort study. Cancer Epidemiol 2017;48:124-30. doi:10.1016/j.canep.2017.04.015

17 Nguyen T, Khan A, Liu Y, et al. The Association Between Statin Use After Diagnosis and Mortality Risk in Patients With Esophageal Cancer: A Retrospective Cohort Study of United States Veterans. Am J Gastroenterol 2018;113:1310-8. doi:10.1038/s41395-018-0169-6

18 Lacroix O, Couttenier A, Vaes E, et al. Impact of metformin on gastric adenocarcinoma survival: A Belgian population based study. Cancer Epidemiol 2018;53:149-55. doi:10.1016/j.canep.2018.02.001

19 Henau K, Van Eycken E, Silversmit G, et al. Regional variation in incidence for smoking and alcohol related cancers in Belgium. Cancer Epidemiol 2015;39:55-65. doi:10.1016/j.canep.2014.10.009

20 Jegou D, Dubois C, Schillemans V, et al. Use of health insurance data to identify and quantify the prevalence of main comorbidities in lung cancer patients. Lung Cancer 2018;125:238-44. doi:10.1016/j.lungcan.2018.10.002

21 Zhou Z, Rahme E, Abrahamowicz M, et al. Survival bias associated with time-to-treatment initiation in drug effectiveness evaluation: A comparison of methods. Am J Epidemiol Published Online First: 2005. doi:10.1093/aje/kwi307

22 Mei Z, Liang M, Li L, et al. Effects of statins on cancer mortality and progression: A systematic review and meta-analysis of 95 cohorts including 1,111,407 individuals. Int J Cancer 
2017;140:1068-81. doi:10.1002/ijc.30526

23 Nielsen SF, Nordestgaard BG, Bojesen SE. Statin use and reduced cancer-related mortality. $N$ Engl J Med 2012;367:1792-802. doi:10.1056/NEJMoa1201735

24 Gray RT, Coleman HG, Hughes C, et al. Statin use and survival in colorectal cancer: Results from a population-based cohort study and an updated systematic review and meta-analysis. Cancer Epidemiol 2016;45:71-81. doi:10.1016/j.canep.2016.10.004

25 Kim ST, Kang JH, Lee J, et al. Simvastatin plus capecitabine-cisplatin versus placebo plus capecitabine-cisplatin in patients with previously untreated advanced gastric cancer: A doubleblind randomised phase 3 study. Eur $J$ Cancer 2014;50:2822-30. doi:10.1016/j.ejca.2014.08.005

26 Konings IRHM, Van Der Gaast A, Van Der Wijk LJ, et al. The addition of pravastatin to chemotherapy in advanced gastric carcinoma: A randomised phase II trial. Eur J Cancer 2010;46:3200-4. doi:10.1016/j.ejca.2010.07.036

27 Iannelli F, Lombardi R, Milone MR, et al. Targeting Mevalonate Pathway in Cancer Treatment: Repurposing of Statins. Recent Pat Anticancer Drug Discov 2018;13:184-200. doi:10.2174/1574892812666171129141211

28 Brown JC, Meyerhardt JA. Obesity and energy balance in GI cancer. J Clin Oncol 2016;34:4217-24. doi:10.1200/JCO.2016.66.8699

29 Hynes O, Anandavadivelan P, Gossage J, et al. The impact of pre- and post-operative weight loss and body mass index on prognosis in patients with oesophageal cancer. Eur J Surg Oncol 2017;43:1559-65. doi:10.1016/j.ejso.2017.05.023

30 Sun P, Chen C, Zhang F, et al. Combined heavy smoking and drinking predicts overall but not disease-free survival after curative resection of locoregional esophageal squamous cell carcinoma. Onco Targets Ther 2016;9:4257-64. doi:10.2147/OTT.S104182

31 Lennon H, Sperrin M, Badrick E, et al. The Obesity Paradox in Cancer: a Review. Curr Oncol Rep 2016;18:56. doi:10.1007/s11912-016-0539-4

32 Lagergren J, Andersson G, Talbäck M, et al. Marital status, education, and income in relation to the risk of esophageal and gastric cancer by histological type and site. Cancer 2016;122:207-12. doi:10.1002/cncr.29731 
33 Emilsson L, García-Albéniz X, Logan RW, et al. Examining bias in studies of statin treatment and survival in patients with cancer. JAMA Oncol 2018;4:63-70. doi:10.1001/jamaoncol.2017.2752

34 Targownik LE, Suissa S. Understanding and avoiding immortal-time bias in gastrointestinal observational research. Am J Gastroenterol 2015;110:1647-50. doi:10.1038/ajg.2015.210 